\title{
Quasiparticles in the bosonization theory of interacting fermion liquids at arbitrary dimensions
}

\author{
Tai-Kai Ng \\ Department of Physics, Hong Kong University of Science and Technology, Clear Water Bay Road, Kowloon, Hong Kong
}

(Received 2 April 2003; published 14 July 2003)

\begin{abstract}
In the bosonization theory, we propose in this paper a definition of "quasiparticles" for interacting fermions at arbitrary space dimenions. In dimensions higher than one, we show that the constructed quasiparticles are consistent with quasiparticle descriptions in the Landau-Fermi-liquid theory, whereas in one dimension the quasiparticles are nonperturbative objects (spinons and holons) obeying fractional statistics. Application of our theory to Fermion liquids with singular Landau interaction is discussed.
\end{abstract}

DOI: 10.1103/PhysRevB.68.041101

The concept of quasiparticles, as excitations adiabatically connected to excitations in corresponding noninteracting Fermi gas, has formed the basis of our understanding of Fermi liquids at dimensions higher than one. ${ }^{1}$ Based on Bethe-ansatz solutions ${ }^{2}$ of exactly solvable models, it is now understood that similar to fermions in higher dimensions, the low energy behaviors of interacting fermions in one dimension can also be described as gases of "free" particles, or quasiparticles, except that these particles are not adiabatically connected to free fermions as in the Landau-Fermiliquid theory, but are nonperturbative objects called "spinon" and "holon," characterizing the spin and charge degrees of freedom of the system separately. ${ }^{3}$ The spinons and holons are neither fermions or bosons, but are objects obeying fractional(exclusion) statistics. ${ }^{4}$

The objective of the present paper is to show that the two rather different pictures of quasiparticles in the LandauFermi-liquid theory and in the Bethe-ansatz solutions in one dimension can be understood in the bosonization theory by proposing a definition of quasiparticle operators. Our approach provides a simple way of unifying the two quasiparticle pictures and provides a more general framework of understanding quasiparticles in interacting fermion systems. We shall also discuss the situation of generalized Fermi liquids with singular Landau interaction within our framework.

To start we first review briefly the bosonization theory in one dimension ${ }^{5}$ and in higher dimensions. ${ }^{6-8}$ The main idea of the bosonization theory is that the low-energy excitation spectrum of Fermi and Luttinger liquids can both be described by effective harmonic theories describing elastic deformation of Fermi surfaces. ${ }^{7,8}$ The deformation at position $\vec{k}_{F}$ on the Fermi surface is described by the coarse-grained Wigner operator ${ }^{8}$

$$
\rho_{\vec{k}_{F} \sigma}(\vec{q})=\frac{1}{V} \sum_{-\Lambda<k<\Lambda} c_{\vec{k}_{F}+k \hat{k}_{F}+\vec{q} / 2 \sigma}^{\dagger} c_{\vec{k}_{F}+k \hat{k}_{F}-\vec{q} / 2 \sigma}
$$

where $V$ is the volume, $\hat{k}_{F}$ is the unit vector along the direction of $\vec{k}_{F}$, and $\Lambda \ll k_{F}$ is the high-momentum cutoff. $c\left(c^{\dagger}\right)$ are the usual fermion annihilation(creation) operators. We set $\hbar=1$ in this paper. Notice that in one dimension $\vec{k}_{F} \rightarrow \pm k_{F}$ and $\rho_{\vec{k}_{F} \sigma}(\vec{q}) \rightarrow \rho_{L(R) \sigma}(q)$, i.e., left and right traveling fermions. $\rho_{\vec{k}_{F}} \sigma(\vec{q})$ 's obey approximate commutation relations ${ }^{7,8}$
PACS number(s): 71.27.+a, 74.25.-q, 11.15.-q

$$
\begin{aligned}
& {\left[\rho_{\vec{k}_{F} \sigma}(\vec{q}), \rho_{\vec{k}_{F}^{\prime} \sigma^{\prime}}\left(-\vec{q}^{\prime}\right)\right]} \\
& \quad=-\delta^{D}\left(\vec{q}-\vec{q}^{\prime}\right) \delta^{D-1}\left(\vec{k}_{F}-\vec{k}_{F}^{\prime}\right) \delta_{\sigma \sigma^{\prime}} \frac{\vec{q} \cdot \vec{k}_{F}}{m} N_{\Lambda}(0),
\end{aligned}
$$

where $D$ is the dimension and $N_{\Lambda}(0)=N(0) / S_{d}$, where $N(0)$ is the density of states of spin- $\sigma$ fermions on the Fermi surface, and $S_{d}=\int d \Omega$ is the solid angle on the Fermi surface. Rigorously speaking, the commutation relation (1) is valid only if the Fermi surface is divided into patches of area $\sim \Lambda^{D-1}$ around each Fermi momentum $\vec{k}_{F}$ (Refs. 7 and 8) and the Fermi momentum $\vec{k}_{F}$ has a resolution of the order of $\Lambda$. This should be kept in mind in the following analysis.

For computation purpose it is convenient to use canonical boson operators $b^{\dagger}(b)$ defined by

$$
\begin{aligned}
\rho_{\vec{k}_{F}}(\vec{q})= & \left(\left|\vec{k}_{F} \cdot \vec{q}\right| / m N_{\Lambda}(0)\right)^{1 / 2}\left[\theta\left(\vec{k}_{F} \cdot \vec{q}\right) b_{\vec{k}_{F} \sigma}^{\dagger}(\vec{q})\right. \\
& \left.+\theta\left(-\vec{k}_{F} \cdot \vec{q}\right) b_{\vec{k}_{F} \sigma}(-\vec{q})\right] .
\end{aligned}
$$

The $b\left(b^{\dagger}\right)$ operators satisfy canonical boson commutation relations. $^{8}$

The low-energy physics of the systems is described by effective Hamiltonian $H=H_{0}+H_{1}$, where $H_{0}$ is the kinetic energy and $H_{1}$ is the interaction. In terms of $b\left(b^{\dagger}\right)$ operators, $H_{0}$ takes the form ${ }^{8}$

$$
H_{0}=\frac{1}{V} \sum_{\vec{k}_{F}, \vec{q}, \sigma} \frac{\left|\vec{k}_{F} \cdot \vec{q}\right|}{m} b_{\vec{k}_{F} \sigma}^{+}(\vec{q}) b_{\vec{k}_{F} \sigma}(\vec{q}),
$$

where we have linearized the fermion dispersion around the Fermi surface to obtain Eq. (2). $H_{1}$ can be written as

$$
H_{1}=\frac{1}{2 V} \sum_{\vec{k}_{F}, \sigma, \vec{k}_{F}^{\prime}, \sigma^{\prime},|\vec{q}|<\Lambda} f_{\vec{k}_{F} \sigma \vec{k}_{F}^{\prime} \sigma^{\prime}}(\vec{q}) \rho_{\vec{k}_{F} \sigma}(\vec{q}) \rho_{\vec{k}_{F}^{\prime} \sigma^{\prime}}(-\vec{q}),
$$

where $f_{\vec{k}_{F} \sigma \vec{k}_{F}^{\prime} \sigma^{\prime}}(\vec{q})$ 's are effective parameters characterizing the residual (marginal) fermion-fermion interactions in the low-energy and the small wave-vector limit., ${ }^{8,9}$ With Eqs. (1)-(3), we obtain the Heisenberg equation of motion for $\rho_{\vec{k}_{F}} \sigma(\vec{q})$ (Ref. 8), 


$$
\begin{aligned}
& \left(i \frac{\partial}{\partial t}+\frac{\vec{k}_{F} \cdot \vec{q}}{m}\right) \rho_{\vec{k}_{F} \sigma}(\vec{q}) \\
& \quad=-\frac{\vec{k}_{F} \cdot \vec{q}}{m} N_{\Lambda}(0) \sum_{\vec{k}_{F}^{\prime} \sigma^{\prime}} f_{\vec{k}_{F} \sigma \vec{k}_{F}^{\prime} \sigma^{\prime}}(\vec{q}) \rho_{\vec{k}_{F}^{\prime} \sigma^{\prime}}(\vec{q}),
\end{aligned}
$$

which is identical to the Landau transport equation for quasiparticles in the Fermi-liquid theory in the $\vec{q} \rightarrow 0$ limit, where $f_{\vec{k}_{F} \sigma \vec{k}_{F}^{\prime} \sigma^{\prime}}(\vec{q} \rightarrow 0)$ can be identified as Landau parameters. ${ }^{8,9}$ The same form of equation of motion for $\rho_{L(R) \sigma}(q)$ is also obtained in one dimension.

Although the equations of motion have the same form in one dimension and in higher dimensions, the resulting eigenstate spectra are very different. At higher dimensions, there are two kinds of eigenstate solutions to Eq. (4), the particlehole continuum and collective modes. ${ }^{10}$ The particle-hole continuum are eigenstates adiabatically connected to the particle-hole spectrum of noninteracting fermions, whereas collective modes are nonperturbative eigenstates that disappear in the absence of interaction. The validity of the Fermiliquid theory is warranted by the existence of adiabatic particle-hole spectrum. ${ }^{10}$ In one dimension, only nonperturbative collective density and spin-wave excitations exist. ${ }^{5}$ The collective excitations are not adiabatically connected to the excitation spectrum of noninteracting fermions, showing that the systems are not Landau-Fermi liquids.

Next, we consider the construction of quasiparticle operators. Our goal is to search for a bosonization representation of the quasiparticle operator that is valid in the low-energy, long-wavelength limit. To gain insight, we first consider noninteracting fermions. In the spirit of the Landau-Fermiliquid theory, we consider a $(\operatorname{spin}-\sigma)$ quasiparticle wave packet with momentum $\vec{k}_{F}$ at position $\vec{r}$. Notice that quantum mechanics requires that there exist uncertainties $\delta r$ $\sim \Lambda^{-1}$ and $\delta k \sim \Lambda$ in the position and momentum of the particle. For noninteracting fermions, the quasiparticle wave packet can be identified as a free $\operatorname{spin}-\sigma$ fermion wave packet described by

$$
\psi_{\vec{k}_{F} \sigma}(\vec{r}) \sim \frac{1}{2 \pi} \int_{-\Lambda}^{\Lambda} k^{D-1} d k e^{-i k \hat{k}_{F} \cdot \vec{r}} \vec{k}_{F}+k \hat{k}_{F} \sigma,
$$

where we have introduced no explicit uncertainty in the $d i$ rection $\hat{k}_{F}$. Uncertainty in direction of the order of $\delta k_{F}$ $\sim \Lambda$ exists implicitly when we divide the Fermi surface into small patches. In the limit $\Lambda \ll k_{F}$, it is easy to see that the wave-packet operator satisfies the equation of motion

$$
\begin{aligned}
i \frac{\partial}{\partial t} \psi_{\vec{k}_{F} \sigma}(\vec{r}) & =\left[\psi_{\vec{k}_{F} \sigma}(\vec{r}), H_{o}-\mu N\right] \\
& =\frac{i \vec{k}_{F} \cdot \vec{\nabla}}{m} \psi_{\vec{k}_{F} \sigma}(\vec{r})+O\left(\Lambda / k_{F}\right),
\end{aligned}
$$

where $N$ is the number of particles and $\mu$ is the chemical potential.

We shall now show that we can use Eq. (5), which specifies the dynamics of a free-particle wave packet, as the defi- nition of quasiparticle operators for free fermions in the bosonization theory. To see this, we write $\psi_{k_{F} \sigma}(\vec{r})$ $=\hat{f}_{\hat{k}_{F}} e^{J_{k_{F}} \sigma^{(\vec{r})}}$, where $J_{\vec{k}_{F} \sigma}(\vec{r}) \sim \sum_{\vec{q}} \alpha_{\vec{k}_{F} \sigma}(\vec{q}, \vec{r}) \rho_{\vec{k}_{F} \sigma}(\vec{q})$ is a linear combination of $\rho_{\vec{k}_{F}}(\vec{q})$ operators, i.e., $\psi_{\vec{k}_{F} \sigma}(\vec{r})$ represents a coherent state of bosonic waves in the bosonization theory. $\hat{f}_{\hat{k}_{F}}$ is an operator (the Klein factor) introduced to ensure the anticommunication relation between $\psi_{k_{F}} \sigma(\vec{r})$ fields in different directions $\vec{k}_{F}$, s. ${ }^{6}$ The linear coefficients $\alpha$ 's characterizing $J_{\vec{k}_{F}} \sigma(\vec{r})$ are determined by the condition that $\psi_{\vec{k}_{F} \sigma}(\vec{r})$ satisfies the equation of motion (5) with the bosonized $H_{0}$ [Eq. (2)]. After some straightforward algebra, we obtain

$$
J_{\vec{k}_{F} \sigma}(\vec{r}) \sim-\left(e^{*}\right) \frac{1}{V} \sum_{\vec{q}} \frac{m e^{-i \vec{q} \cdot \vec{r}}}{N_{\Lambda}(0) \vec{k}_{F} \cdot \vec{q}} \rho_{\vec{k}_{F} \sigma}(\vec{q}),
$$

where $e^{*}$ is an arbitrary number that is not fixed by the equation of motion. The significance of $e^{*}$ can be determined by examining the communication relation between $\psi$ operator and the total charge operator $\rho(\vec{q})$ $=\sum_{\vec{k}_{F}, \sigma} \rho_{\vec{k}_{F} \sigma}(\vec{q})$. We obtain

$$
\left[\psi_{\vec{k}_{F} \sigma}(\vec{r}), \rho(-\vec{q})\right]=\left(e^{*}\right) e^{-i \vec{q} \cdot \vec{r}} \psi_{\vec{k}_{F} \sigma}(\vec{r}),
$$

showing that $e^{*}$ represents the charge carried by the quasiparticle and is equal to one for free fermions. The reason why we cannot determine $e^{*}$ by the equation of motion is that the solutions of Eq. (5) represent coherent superposition of boson waves traveling with constant velocity $\vec{v}_{F}=\vec{k}_{F} / m$, and superposition of different solutions is again a solution to the equation. We note that in the usual bosonization theory, $\psi_{\sigma}(\vec{r})=\sum_{\vec{k}_{F}} \psi_{\vec{k}_{F} \sigma}(\vec{r})$ is identified as the bosonization representation of fermion operator and is usually derived by the condition that $\psi_{\sigma}(\vec{r})$ satisfies the correct commutation relation with the density operator. ${ }^{6,8}$ We define $\psi_{\sigma}(\vec{r})$ as the quasiparticle operator through the equation of motion in our approach. For free fermions, the two definitions give identical result.

In the presence of interaction, we define quasiparticle operators as operators representing coherent states of bosonic waves, satisfying equations of motion of the form

$$
\begin{aligned}
i \frac{\partial}{\partial t} \psi_{k_{F} \gamma}^{(Q)}(\vec{r}) & =\left[\psi_{\vec{k}_{F} \gamma}^{(Q)}(\vec{r}), H-\mu N\right] \\
& \sim i \vec{v}_{\gamma} \cdot \vec{\nabla} \psi_{\vec{k}_{F} \gamma}^{(Q)}(\vec{r})+O\left(\Lambda / k_{F}\right),
\end{aligned}
$$

where $H$ is the full bosonized Hamiltonian and $\gamma$ is the branch index. $\vec{v}_{\gamma} \sim \vec{k}_{F} / m_{\gamma}$ is the velocity of branch $\gamma$ quasiparticles at position $\vec{k}_{F}$ on the Fermi surface. The nature of the different branches and corresponding $\vec{v}_{\gamma}$ is determined self-consistently by the condition that $\psi_{\vec{k}_{F} \gamma}^{(Q)}(\vec{r})$ satisfies the equation of motion (7). Writing $\psi_{\vec{k}_{F}}^{(Q)}(\vec{r})=\hat{f}_{\hat{k}_{F}} e^{J_{\vec{k}_{F}}^{(Q)}(\gamma, \vec{r})}$, 
where $J_{\vec{k}_{F} \sigma}^{(Q)}(\vec{r}) \sim \sum_{\vec{q}} \alpha_{\vec{k}_{F} \sigma}(\vec{q}, \vec{r}) \rho_{\vec{k}_{F} \sigma}(\vec{q})$ as before, we obtain from Eq. (7) a linear eigenvalue equation for the coefficients $\alpha$ 's. The nature of the quasiparticle branches and $\vec{v}_{\gamma}$ are determined by the eigenvectors and eigenvalues of the eigenvalue equation.

To illustrate this, we consider interacting fermions at dimensions $D>1$. An easy way to obtain the quasiparticle operators is to observe that at dimenions higher than one, a continuous spectrum of a particle-hole pair solution $\vec{\rho}_{\vec{p}_{F} s}(\vec{q})=\rho_{\vec{p}_{F} s}(\vec{q})+\sum_{\vec{k}_{F} \sigma} \xi_{\vec{p}_{F} s \vec{k}_{F} \sigma}(\vec{q}) \rho_{\vec{k}_{F}}(\vec{q})$ to Eq. (4) exists, with eigenenergy $\epsilon=\vec{p}_{F} \cdot \vec{q} / m$ and

$$
\xi_{p_{F} s \vec{k}_{F} \sigma}(\vec{q})=\frac{1}{V}\left(\begin{array}{c}
\frac{\vec{p}_{F} \cdot \vec{q}}{m} \\
\frac{\vec{k}_{F} \cdot \vec{q}}{m}-\frac{\vec{p}_{F} \cdot \vec{q}}{m}
\end{array}\right) N_{\Lambda}(0) A_{\vec{k}_{F} \sigma \vec{p}_{F} s}(\vec{q}),
$$

where $A_{\vec{k}_{F} \sigma \vec{p}_{F} s}(\vec{q})$ is the quasiparticle scattering matrix in the Landau-Fermi-liquid theory. ${ }^{8,10}$ A quasiparticle operator with momentum $\vec{p}_{F}, \psi_{p_{F} s}^{(Q)}(\vec{r})=\hat{f}_{\hat{p}_{F}} e^{J \vec{p}_{F}}(s, \vec{r})$, satisfying the equation of motion (7) can be obtained by choosing

$$
J_{\vec{p}_{F}}^{(Q)}(s, \vec{r})=-\frac{1}{V} \sum_{\vec{q}} \frac{m e^{-i \vec{q} \cdot \vec{r}}}{N_{\Lambda}(0) \vec{p}_{F} \cdot \vec{q}} \vec{\rho}_{\vec{p}_{F}} \sigma(\vec{q}) .
$$

The branch index is given by $\gamma=s$, i.e., spin index and $m_{\gamma}$ $=m$ as for noninteracting fermions. The quasiparticle operators constructed in this way represent bare fermions dressed by particle-hole pair excitations (described by the coefficients $\xi$ ) and are adiabatically connected to the noninteracting fermions. Notice that we have set $e^{*}=1$ to ensure adiabaticity. We expect that the quasiparticle operator constructed in this way corresponds to the eigenquasiparticle state in the Fermi-liquid theory. ${ }^{10}$ To confirm this we compute the charge carried by the quasiparticle operator we constructed. We obtain

$$
\left[\psi_{\vec{p}_{F} s}^{Q}(\vec{r}), \rho(-\vec{q})\right]=\left(1-\sum_{\vec{k}_{F} \sigma} \xi_{\vec{k}_{F} \sigma \vec{p}_{F} s}(\vec{q})\right) e^{-i \vec{q} \cdot \vec{r}} \psi_{\vec{p}_{F} s}^{Q}(\vec{r}),
$$

suggesting that the charge $\langle\rho(\vec{q})\rangle$ carried by the quasiparticle excitation is $\langle\rho(\vec{q})\rangle=1-\sum_{\vec{k}_{F} \sigma} \xi_{\vec{k}_{F} \sigma \vec{p}_{F} s}(\vec{q})$, in exact agreement with the Fermi-liquid theory where the $\xi$ factors describe the screening effect. ${ }^{10}$ The same agreement with the Fermi-liquid theory is also obtained for the current and spin operators.

Next, we consider fermions in one dimension. We consider bosonized Hamiltonian $H$ with $\vec{k}_{F} \rightarrow \pm k_{F}=R, L$ and $f_{\vec{k}_{F} \sigma \vec{k}_{F}^{\prime} \sigma^{\prime}}(\vec{q})=f_{s}(q)+f_{a}(q) \vec{\sigma} \cdot \vec{\sigma}^{\prime}$, i.e., we neglect currentcurrent interactions. The resulting equation of motion (4) for bosonic excitations has two branches of solution with dispersions $\boldsymbol{\epsilon}_{s(a)}(q)=\left[\sqrt{v_{F}^{2}+2 f_{s(a)}(q) v_{F} / \pi}\right] q=v_{s(a)} q$, where $v_{F}=k_{F} / m$ and $s$ and $a$ represents density and spin-wave fluctuations, respectively. ${ }^{5}$ Notice that $v_{s} \neq v_{a}$ as long as $f_{s}(q) \neq f_{a}(q)$, reflecting general spin-charge separation of interacting fermions in one dimension.

To construct quasiparticle operators we proceed as before and define quasiparticle operators using Eq. (7), with $\psi_{p_{F} \gamma}^{(Q)}(\vec{r}) \rightarrow \psi_{L(R) \gamma}^{(Q)}(x)$ and $\left(\vec{k}_{F} \cdot \vec{\nabla}\right) / m \rightarrow \pm v_{F} \partial / \partial x$. Writing $\psi_{L(R) \gamma}^{(Q)}(x)=\hat{f}_{L(R)} e^{J_{L(R)}^{(Q)}(\gamma, x)}$, where $J^{(Q)}$ is linear in the $\rho_{L(R) \sigma}(\vec{q})$ operators, we obtain from solving Eq. (7),

$$
\begin{aligned}
J_{R(L)}^{(Q)}(\gamma, x)= & \frac{e_{\gamma}^{*}}{2 V} \sum_{q} \frac{e^{-i q x}}{q}\left(\left(\frac{+(-) 1}{\eta_{\gamma}}\right)\left[\rho_{L \gamma}(q)+\rho_{R \gamma}(q)\right]\right. \\
& \left.+\left[\rho_{R \gamma}(q)-\rho_{L \gamma}(q)\right]\right)
\end{aligned}
$$

where $\gamma=s, a$ and $\rho_{L(R)[s(a)]}(q)=\Sigma_{\sigma}[1(\sigma)] \rho_{L(R) \sigma}(q)$ is the coarse-grained density (spin) Wigner operator. Notice that $\psi_{L(R) s(a)}^{(Q)}(x)$ 's constructed in this way represent quasiparticles corresponding to coherent states formed by density $s$ and spin $a$ bosonic waves separately (spin-charge separation) and are not adiabatically connected to the original fermions. The self-determined quasiparticle velocities $v_{s(a)}$ are equal to the corresponding boson density (spin) wave velocities and $\eta_{s(a)}=v_{F} / v_{s(a)}$. The magnitude of charge (spin) carried by the quasiparticle $e_{s(a)}^{*}$ can be determined if we identify the quasiparticle excitations we constructed as holons $(\gamma=s)$ and spinons $(\gamma=a)$ in Bethe-ansatz solutions. The charge (spin) carried by the holon (spinon) equals $1(1 / 2)$ and $e_{s}^{*}=e_{a}^{*}=1$.

It is interesting to point out that the operator $\psi_{L(R) \gamma}^{(Q)}(x)$ has been introduced as the bosonization representation of particles with fractional (exclusion) statistics. ${ }^{11}$ The statistics of the quasiparticles can be determined directly by examining the commutation rules between quasiparticle operators. It is easy to show that

$\psi_{L(R) \gamma}^{(Q)}(x) \psi_{L(R) \gamma}^{(Q)}\left(x^{\prime}\right)=e^{\left(i \pi / \eta_{\gamma}\right) \operatorname{sgn}\left(x-x^{\prime}\right)} \psi_{L(R) \gamma}^{(Q)}\left(x^{\prime}\right) \psi_{L(R) \gamma}^{(Q)}(x)$

indicating that the charge and spin quasiparticles are exclusons with statistical parameter $1 / \eta_{s}$ and $1 / \eta_{a}$, respectively. ${ }^{4,11}$ Our definition of quasiparticles identify these operators as quasiparticles in one-dimensional fermionic systems naturally.

A natural question that arises is what is the most general criteria by which quasiparticle operators can be constructed in a fermionic system at dimensions $>1$, given that the lowenergy dynamics of the system is described by the equation of motion of form (4)? This question is important because it has been observed in recent years that non-Fermi-liquid behavior appears in certain systems at two dimensions, for example, fermions in the half-filled Landau level or in strongly fluctuating gauge fields. ${ }^{12,13}$ The low-energy physics of these systems are believed to be described by transport equation similar to Eq. (4), except that the effective Landau interactions and corresponding effective masses are frequency dependent and are singular in the $\omega, q \rightarrow 0$ limit. ${ }^{12,13}$

For frequency-independent Landau interactions, it can be shown that quasiparticle operators defined by Eq. (7) can be 
constructed as long as there exists a continuum eigenspectrum of the equation of motion (4) with eigenenergy $\epsilon_{\vec{p}_{F}}(\vec{q})=\vec{v}_{\gamma} \cdot \vec{q}$ at small $q$, at every point $\vec{p}_{F}$ on the Fermi surface. The solutions may or may not be adiabatically connected to the noninteracting fermions. The physical reason for this requirement is clear: if the low-energy excitations of the system are characterized by quasiparticle occupation numbers with quasiparticle dispersion that is continuous across the Fermi surface, then particle-hole spectrum of the form $\epsilon_{p_{F}}(\vec{q})=\vec{v}_{\gamma} \cdot \vec{q}$ must exist for all $\vec{p}_{F}$ at small $q$. What is surprising is that this is also a sufficient condition for the existence of quasiparticles in the bosonization theory with our definition.
For transport equations with frequency-dependent Landau parameters, the above conclusion does not hold because the eigenstates of the transport equations are not orthogonal to each other, although we expect that the conclusion may be qualitatively similar. This seems to be the case for fermions in the half-filled Landau level or in fluctuation transverse gauge fields. ${ }^{12}$ Notice that in the case when the particle-hole continuum solution is nonperturbative, we expect that some Ward identities derived for Landau-Fermi liquids may be violated, and non-Fermi-liquid behaviors may appear in the physical response functions.

The author thanks Professor Y. B. Kim for helpful comments.
${ }^{1}$ L.D. Landau, Sov. Phys. JETP 3, 920 (1956); 8, 70 (1959).

${ }^{2}$ See, e. g., B. Sutherland, in Exactly Solvable Problems in Condensed Matter and Relativistic Field Theory, edited by B. S. Shastry, S. S. Jha, and V. Singh, Lecture Notes in Physics Vol. 242 (Springer, Berlin, 1985), p. 1.

${ }^{3}$ M. Ogata and H. Shiba, Phys. Rev. B 41, 2326 (1990).

${ }^{4}$ F.D.M. Haldane, Phys. Rev. Lett. 67, 937 (1991); Y. Hatsugai, M. Kohmoto, T. Koma, and Y.S. Wu, Phys. Rev. B 54, 5358 (1996).

${ }^{5}$ S. Tomonaga, Prog. Theor. Phys. 5, 544 (1950); F.D.M. Haldane, J. Phys. C 14, 2585 (1981).

${ }^{6}$ A. Luther, Phys. Rev. B 19, 320 (1979).

${ }^{7}$ F. D. M. Haldane, in Luttinger's Theorem and Bosonization of the Fermi Surface, Proceedings of the International School of Physics “Enrico Fermi," Course CXXI, Varenna, 1992, edited by R.
Schrieffer and R. A. Broglia (North-Holland, New York, 1994).

${ }^{8}$ A. Houghton and J.B. Marston, Phys. Rev. B 48, 7790 (1993); A.H. Castro Neto and E. Fradkin, Phys. Rev. Lett. 72, 1393 (1994).

${ }^{9}$ R. Shankar, Rev. Mod. Phys. 66, 129 (1994).

${ }^{10}$ P. Nozières and D. Pines, in The Theory of Quantum Liquids, edited by D. Pines, Advanced Book Classics (Perseus Books, Cambridge, 1996).

${ }^{11}$ Y.S. Wu and Y. Yu, Phys. Rev. Lett. 75, 890 (1995).

${ }^{12}$ Y.B. Kim, P.A. Lee, and X.G. Wen, Phys. Rev. B 52, 17275 (1995); A. Stern and B.I. Halperin, ibid. 52, 5890 (1995).

${ }^{13}$ P.A. Bares and X.G. Wen, Phys. Rev. B 48, 8636 (1993); Y.L. Liu and T.K. Ng, Phys. Rev. Lett. 83, 5539 (1999). 